\title{
Non-Operative Management of Pilonidal Sinuses Located Around Anus
}

\author{
Kemal Arslan $^{1^{*},}$ Osman Doğru' ${ }^{1}$, Erhan Aygen ${ }^{2}$, Ersin Turan ${ }^{1}$ \\ ${ }^{1}$ Department of General Surgery, Konya Training and Research Hospital, Konya, Turkey \\ ${ }^{2}$ Department of General Surgery, Medical Faculty of Firat University, Elazı $\breve{g}$, Turkey \\ Email: "arslanka74@hotmail.com
}

Received October 3, 2012; revised November 6, 2012; accepted November 16, 2012

\begin{abstract}
Aim: Pilonidal disease is generally located at sacrococcygeal region whereas it is rarely located near anus. The aim of this study is to discuss the results of crystallized phenol application that we performed for patients with sinus pilonidalis located near anus with 95\% success rate. Patients and Methods: Patients admitted between 2005 to 2011 with sinuses located in $2 \mathrm{~cm}$ range of anus or were primarily located up to $2 \mathrm{~cm}$ to the anal verge were enrolled in the study. Patients' demographic features, Body Mass İndex (BMI), family history, skin color, hair thickness, number of sinus openings, and the status of the sinus (acute vs. chronic) were recorded. Crystallized phenol was applied into the sinus. The patients were followed-up after recovery during the first 6 months and annually afterwards. Results: A total of 25 sinus pilonidalis cases located near anus were encountered. All patients were male; crystallized phenol application was performed on all patients a total of 115 times. The mean number of applications was 5.6 (between 4 and 8 times). The mean recovery period was 74.5 days (range: $31-154$ ) and the mean follow-up period was 36.16 months (range: 18 - 48). No surgical intervention was required. Conclusion: Sinus pilonidalis cases located in perianal region can be successfully treated with the crystallized phenol application which is a simple and inexpensive method, that can easily be performed in an outpatient setting.
\end{abstract}

Keywords: Pilonidal Sinus around Anus; Non-Opretive Treatment; Crystallized Phenol

\section{Introduction}

Most of the reported sinus pilonidalis cases are found in the sacrococcygeal region. In few cases, tracks run towards the caudal direction and may open lateral or even anterior of the anus [1-3], and cause confusion in the diagnosis and treatment. The differential diagnosis should include perianal fistula. After exclusion of the fistula, surgical treatment is the generally preferred choice of treatment. Despite the successful results of new techniques, some complex cases such as patients with secondary openings away from the midline can lead some problems. Total subcutaneous fistulectomy plus Karydakis flap, developed by Kulacoglu et al., is an effective surgical modality for this complicated patients [4]. Although successful results have been reported, surgical intervention in this region has always been a challenge under these circumstances. Thus, management using the conservative methods is favored by patients. The aim of this study was to discuss the results of crystallized phenol applications that we performed in sinus pilonidalis located near to anus with a $95 \%$ success rate [5] in such challenging patients.

${ }^{*}$ Corresponding author.

\section{Patients and Methods}

Of the 35 patients who were admitted to our clinic between 2005 to 2011 with sinus pilonidalis located near anus, 25 were enrolled in this study. The others refused to attend the study and were operated. The sinuses were at a distance of $2 \mathrm{~cm}$ to the anal verge in the caudal direction or were primarily located up to $2 \mathrm{~cm}$ to the anal verge in the perianal region. All patients were performed rectoscopy and other pathologies that may cause similar lesions in the perianal region were all excluded. Patients with isolated sinus pilonidalis were only included in this study. The patients' demographic datas, Body Mass Index (BMI), family history, skin color and hairystatus, number of sinus openings, and the status of the sinus (acute vs. chronic) were recorded. The patients were asked to shave the intervention area and told to keep clean without hair and crystallized phenol was placed into the sinus as described before [4]. The patient was placed in a prone position and the area including the sinus opennings was shaved. Sinus openings less than $3 \mathrm{~mm}$ in diameter were widened after local anesthesia. The anus was protected with a swab or cotton wool, and the rest of the area was iberally coated with nitrofurantoin ointment (Furacin, 
acıbası Ilac, San ve Tic A.S., Istanbul, Turkey). Crystallized phenol (Botapharma Laboratories, Ankara, Turkey) was applied, waited for approximately 2 minutes, and then was teken out by applying pressure on the sinus tract. This procedure was repeated 2 or 3 times depending on the width of the sinus, and the wound was then closed with a gauze pack. When an unopened abscess was noted, the purulent material was drained surgically before hair removal and application of the crystallized phenol. If an abscess had previously been opened, hair removal and crystallized phenol application were performed directly. Patients were allowed to return to their normal daily activities immediately after the procedure. The first follow-up visit took place after 1 week. If the patient's wound had no leakage anymore, no further procedures were undertaken. If leakage from the sinus tract was observed, the treatment procedure was repeated one more time as described above at the second or fourth week after the initial procedure. After the fourth week, patients were followed up at 3-week intervals. When no more leakage from the wound was observed, treatment was terminated. The patients were followed-up once a month after recovery during the first 6 months and annually afterwards. Closure of the orifices was accepted as complete cure. Recurrence was classified as sinus reappearance or abscess formation or leakage following complete cure.

\section{Results}

During the study, a total of 25 sinus pilonidalis patients with perianal location near the anus were noted. All patients were male; the mean age was 27.08 years with a range of 16 to 46 years. The mean BMI was 25.9 (range: 20.5 - 31.4). Out of 25 patients, 7 patients $(28 \%)$ had a positive family history. Of the sinuses, 21 (84\%) were chronic, 4 (16\%) were acute, and the mean number of orifices was 4 (range: $1-8$ ). Seven of the cases $(28 \%)$ were recurrences that had been operated previously and $18(72 \%)$ were primary cases. In acute patients with abscess, the abscesses were drained and crystallized phenol was applied in the same session. In patients without abscess but with purulent discharge and with signs of acute infection, crystallized phenol was applied in the first visit. Although no antibiotics were given in acute cases receiving crystallized phenol, it was observed that signs of infection resolved easily and rapidly. Crystallized phenol applications were performed on 25 patients with a total of 115 times. The mean number of application was 5.6 (range: 4 - 8). The mean recovery period was 74.5 days (range: 31 - 154) and the mean follow-up period was 36.16 months (range: 18 - 48).

During the follow-up, one patient presented again with the complaint of discharge on the second week of the recovery. On examination, few hairs were found in the sinus tract with discharge and two additional sessions of crystallized phenol applications were performed again and the patient had no additional problems during the follow-up. No problems occurred in remained patients during the follow-ups. Surgical intervention was not required in any of these patients.

Rapidly resolving skin erosions around the orifice were observed in two patients due to the phenol overflow. No additional complications developed except for pain. Primary perianal sinus pilonidalis was presented in Figures 1(a) and (b). The pre-procedure and post-pro-

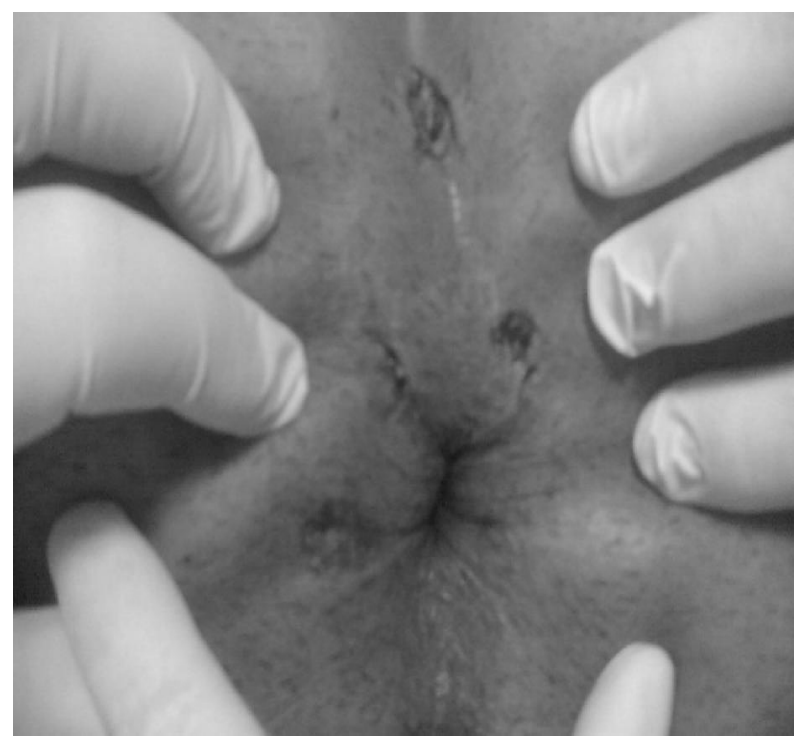

(a)

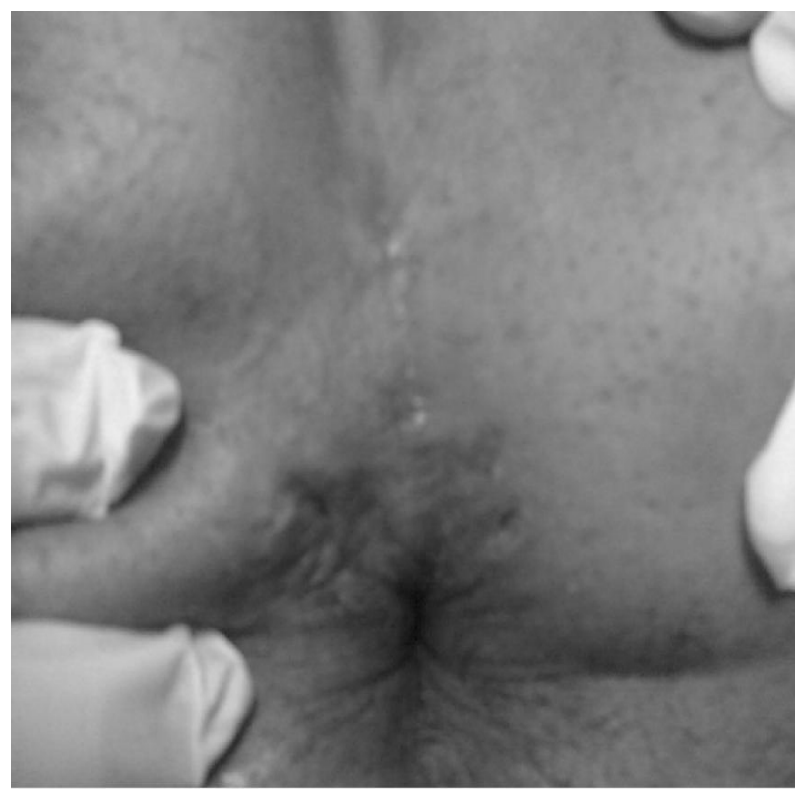

(b)

Figure 1. (a) A patient with sinus pilonidalis located near anus; (b) Patient after healing occurs. 
cedure views of two cases with operated recurrent and a new focus in the perianal region were presented in Figures 2 (a) and (b).

\section{Discussion}

There is limited data in the literature about the treatment of sinus pilonidalis located around the perianal region. Most are presented as case reports [1-2]. There are no large series or considerable experience.Similarly, we could not find any study on the conservative treatment of perianal sinus pilonidalis. Although the number of cases

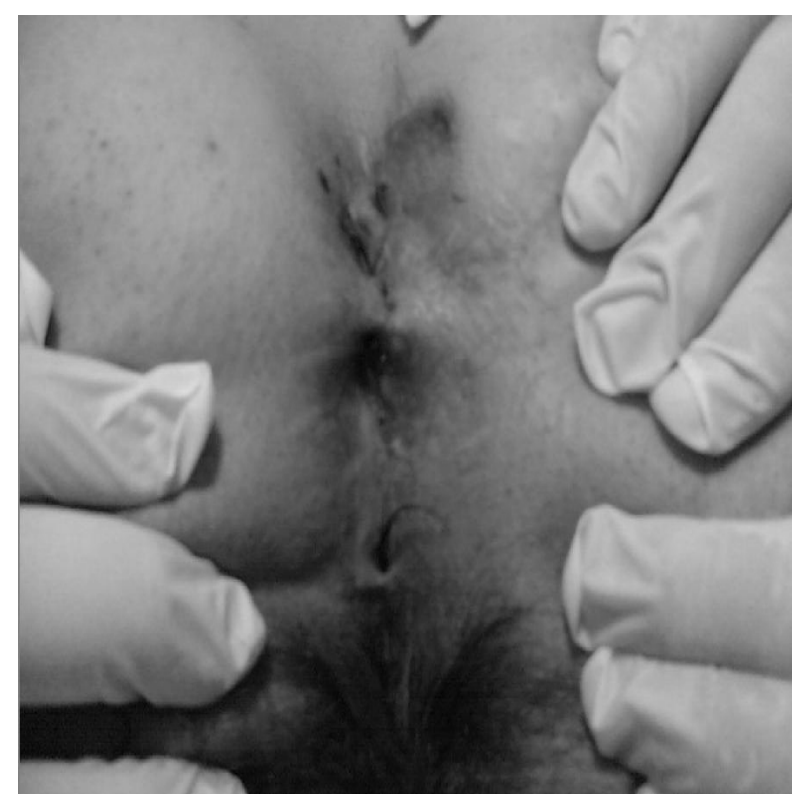

(a)

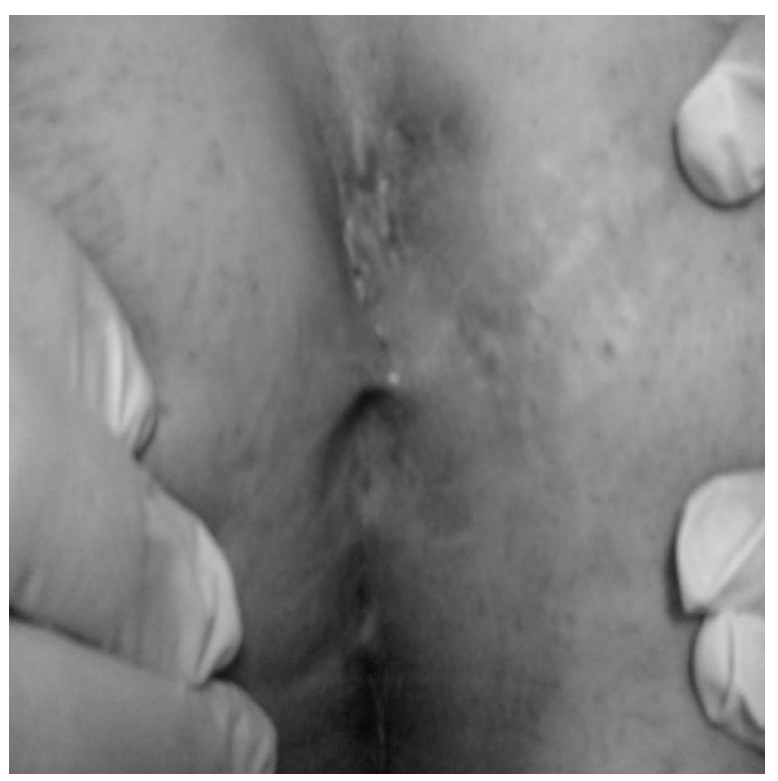

(b)

Figure 2. (a) A patient with sinus pilonidalis located near anus; (b) Patient after healing occurs. was small in our study, we presented here a successful conservative treatment of sinus pilonidalis located in the perianal region and it seems to be the largest patient number in the English Literature.

It is important to diagnose sinus pilonidalis located in the perianal region and to differentiate it from perianal fistulas. The main difference is that pilonidal sinus disease originates subcutaneously, whereas anal fistulas have an enteric communication with the anal canal and the rectum. In case of failure in this differentiation, recurrences are inevitable due to inadequate treatment $[2,6]$.

Rectoscopy and fistulography are the most important two inverventions for the differential of the diagnosis $[1-3,6]$. If a connection between the external orifice and anal canal and/or the rectum is shown with one of these investigations, this is in favor of fistula. In the absence of a connection, it is more likely to be sinus pilonidalis. The treatment plan should be changed according to the diagnosis.

Magnetic Resonance Imaging (MRI) has become a standard modality in the diagnosis and the classification of fistulas and is recommended in suspected and obscured cases in the differential diagnosis of dermoid cysts $[3,6,7]$. In spite of the fact that, sinus pilonidalis is subcutaneous and fistulas are connected with the anal canal or the rectal mucosa, it was observed that in some cases, infection originating from the pilonidal sinus can extent to the inter-sphincteric or even the ischiorectal space and the orifices of perianal fistula may reach to the natal cleft. So, MRI provides valuable information in such cases [8]. We performed rectoscopy to all patients in our study and hydrogen peroxide was administered into the external orifice to be sure that there was no opening into the rectum or in the anal canal. When it is decided that the diagnosis were sinus pilonidalis, the patients were treated with crystrallized phenol. No obscure cases has been noted, therefore, no patients required MRI.

Until recently, surgery was the first-line treatment in perianal sinus pilonidalis. In most case reports, infected tissues were all resected and then left open for secondary healing. It is difficult to comment on the success rates as they were not presented as a study; however, the authors stated that they had experienced no serious problems [1-3]. Total subcutaneous fistulectomy combined with the Karydakis flap was performed on 14 patients with sinus pilonidalis with perianal extension in the study by Kulacoglu et al. They reported a success rate of 100\% [4]. But it can not be classified in non-operative treatment fashion. In our report of 12 cases with crystallized phenol application and with a mean follow-up of 36.16 months, we can consider our success rate as $100 \%$ with conservative treatment. Although it seems that recurrence 
occurred in the early period in one patient, we believe that we misinterpreted the signs of this patient after the first phenol application. As a matter of fact, the discharge appearing after two weeks in this patient was managed with crystallized phenol application again and no additional problems were noted on the follow-up. Crystallized phenol application has no serious complications. We experienced minimal skin erosion and pain in some patients due to phenol overflow on the surrounding skin of the orifices. This treatment did not cause any loss of working day and patients could perform their daily activities without any limitation, after a procedure taking approximately 15 - 20 minutes.

In conclusion, we believe that perianal located sinus pilonidalis can also be successfully treated with the crystallized phenol application. Crystallized phenol application is a simple and inexpensive method that can be performed in an outpatient setting (which decreases the time off work-almost zero). This procedure is suggested as the first-line treatment for patients with any type of pilonidal sinuses.

\section{REFERENCES}

[1] T. H. Walsh and C. V. Mann, "Pilonidal Sinuses of the Anal Canal," British Journal of Surgery, Vol. 70, No. 1, 1983, pp. 23-24. doi:10.1002/bjs. 1800700108
[2] S. Vallance, "Pilonidal Fistulas Mimicking Fistulas-in-Ano," British Journal of Surgery, Vol. 69, No. 3, 1982, pp. 161162. doi:10.1002/bjs. 1800690317

[3] B. A. Taylor and L. E. Hughes, "Circumferential Perianal Pilonidal Sinuses," Diseases of the Colon \& Rectum, Vol. 27, No. 2, 1984, pp. 120-122. doi:10.1007/BF02553991

[4] M. Testini, S. Miniello, B. Di Venere, G. Lissidini and E. Esposito, "Perianal Pilonidal Sinus. Case Report," Annali Italiani di Chirurgia, Vol. 73, No. 3, 2002, pp. 339-341.

[5] H. Kulacoglu, C. Dener, H. Tumer and R. Aktimur, "Total Subcutaneous Fistulectomy Combined with Karydakis Flap for Sacrococcygeal Disease with Secondary Perineal Opening," Colorectal Disease, Vol. 8, No. 2, 2006, pp. 120123. doi:10.1111/j.1463-1318.2005.00872.x

[6] O. Dogru, C. Camci, E. Aygen, M. Girgin and O. Topuz, "Pilonidal Sinus Treated with Crystallized Phenol: An Eight-Year Experience," Diseases of the Colon \& Rectum, Vol. 47, No. 11, 2004, pp. 1934-1938. doi:10.1007/s10350-004-0720-y

[7] W. Alrawashdeh, S. Ajaz, T. M. Hammond, T. R. C. Porrett and P. J. Lunniss, "Primary Anal Pilonidal Disease," Colorectal Disease, Vol. 10, No. 3, 2008, pp. 303-306. doi:10.1111/j.1463-1318.2007.01382.x

[8] S. A. Taylor, S. Halligan and C. I. Bartram, "Pilonidal Sinus Disease: MR İmaging Distinction from Fistula in Ano," Radiology, Vol. 265, No. 3, 2003, pp. 662-667. doi:10.1148/radiol.2263011758 